\title{
Methods in Molecular Biology
}

\author{
Series Editor \\ John M. Walker \\ School of Life Sciences \\ University of Hertfordshire \\ Hatfield, Hertfordshire, AL10 9AB, UK
}

For further volumes:

http://www.springer.com/series/7651 



\section{NanoBiotechnology Protocols}

\section{Second Edition}

Edited by

\section{Sandra J. Rosenthal and David W. Wright}

Department of Chemistry, Vanderbilt University, Nashville, TN, USA 


\section{Editors}

Sandra J. Rosenthal

Department of Chemistry

Vanderbilt University

Nashville, TN, USA

\author{
David W. Wright \\ Department of Chemistry \\ Vanderbilt University \\ Nashville, TN, USA
}

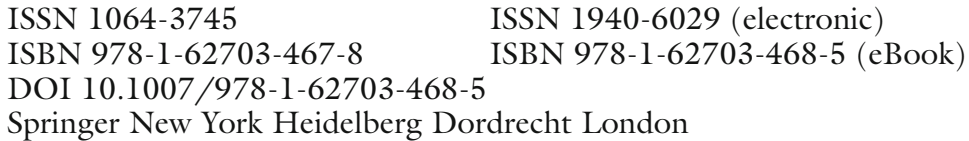

Library of Congress Control Number: 2013938838

\section{(C) Springer Science+Business Media New York 2013}

This work is subject to copyright. All rights are reserved by the Publisher, whether the whole or part of the material is concerned, specifically the rights of translation, reprinting, reuse of illustrations, recitation, broadcasting, reproduction on microfilms or in any other physical way, and transmission or information storage and retrieval, electronic adaptation, computer software, or by similar or dissimilar methodology now known or hereafter developed. Exempted from this legal reservation are brief excerpts in connection with reviews or scholarly analysis or material supplied specifically for the purpose of being entered and executed on a computer system, for exclusive use by the purchaser of the work. Duplication of this publication or parts thereof is permitted only under the provisions of the Copyright Law of the Publisher's location, in its current version, and permission for use must always be obtained from Springer. Permissions for use may be obtained through RightsLink at the Copyright Clearance Center. Violations are liable to prosecution under the respective Copyright Law.

The use of general descriptive names, registered names, trademarks, service marks, etc. in this publication does not imply, even in the absence of a specific statement, that such names are exempt from the relevant protective laws and regulations and therefore free for general use.

While the advice and information in this book are believed to be true and accurate at the date of publication, neither the authors nor the editors nor the publisher can accept any legal responsibility for any errors or omissions that may be made. The publisher makes no warranty, express or implied, with respect to the material contained herein.

Printed on acid-free paper

Humana Press is a brand of Springer

Springer is part of Springer Science+Business Media (www.springer.com) 


\section{Preface}

Nanobiotechnology holds the promise of providing revolutionary insight into aspects of biology ranging from fundamental questions such as elucidating molecular mechanisms of brain disorders to extraordinary applications such as the detection of a single cancer cell in a population of a million cells. Since the publication of the first volume of Nanobiotechnology Protocols 6 years ago, this relatively new field at the intersection of nanoscience and biotechnology has advanced from childhood to adolescence. It is already clear, though, that nanobiotechnologies have found a permanent place in the laboratory. To that end, it is essential that the underlying approaches be based on solid, reproducible methods. It is the goal of NanoBiotechnology Protocols II to provide novice and experienced researchers alike a cross section of the methods employed in two foundational areas of nanobiotechnology: imaging and detection. NBPII also explores new nano-bio constructs and examines the toxicology of nanomaterials.

Nowhere has nanobiotechnology made a more significant impact than in the area of biological imaging and detection. Chapters 1-9 address different nanoprobes and methods for imaging or detection. The substances that make up these probes range from semiconductors to noble metals to carbon and can offer signals several-fold brighter and substantially more stable than traditional organic probes. Additionally, these imaging approaches can be translated to diagnostic approaches for the detection of important pathogens or disease states. Nanobiotechnology is also embracing the interface between hard inorganic materials and the soft functional biology through important designs using amino acids, peptides, and nucleotides (Chapters 10-13). These building blocks afford the research a variety of approaches that not only can be tuned to control the shape, habit, and properties of a nanoparticle but also allow the self assembly of these particles into larger, functional constructs. Finally, in any emerging technology, there are important safety concerns about the materials. This is especially true in an emerging field such as nanobiotechnology, where truly new materials will be interacting in a biological context. For that reason, establishing the toxicology of these materials is an important "next step" in their development and two protocols for toxicity studies are presented in Chapters 14 and 15. Together, these chapters highlight important current areas of development and directions of the field for the future. 



\section{Contents}

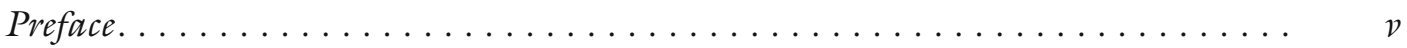

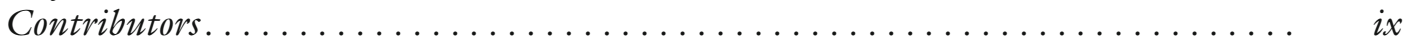

1 High-Aspect-Ratio Gold Nanorods: Their Synthesis and Application to Image Cell-Induced Strain Fields in Collagen Films . . . . . . . . . . . . . . .

Davin J. Chernak, Patrick N. Sisco, Edie C. Goldsmith, Sarab C. Baxter, and Catherine J. Murphy

2 Imaging Intracellular Quantum Dots: Fluorescence Microscopy and Transmission Electron Microscopy . . . . . . . . . . . . . . . .

Craig J. Szymanski, Hong Yi, Joshua L. Liu, Elizabeth R. Wright, and Christine K. Payne

3 Imaging of Cell Populations in Atherosclerosis Using Quantum Dot Nanocrystals . . . . . . . . . . . . . . . . . . . . . . Joshua R. Trantum and Ashwath Jayagopal

4 Imaging of Endothelial Progenitor Cell Subpopulations in Angiogenesis Using Quantum Dot Nanocrystals . . . . . . . . . . . . . . . . . . Joshua M. Barnett, John S. Penn, and Ashwath Jayagopal

5 Imaging Single Synaptic Vesicles in Mammalian Central Synapses with Quantum Dots . . . . . . . . . . . . . . . . . . . . . . . . . . . . . . Qi Zhang

6 Quantum Dot-Based Single-Molecule Microscopy for the Study of Protein Dynamics . . . . . . . . . . . . . . . . . . . . . . . . . . . . . . . Jerry C. Chang and Sandra J. Rosenthal

7 Three-Dimensional Molecular Imaging with Photothermal Optical Coherence Tomography . . . . . . . . . . . . . . . . . . .

Melissa C. Skala, Matthew J. Crow, Adam Wax, and Joseph A. Izatt

8 Detecting Respiratory Syncytial Virus Using Nanoparticle-Amplified Immuno-PCR. . . . . . . . . . . . . . . . . . . . . . . . . . . . .

Jonas W. Perez, Nicholas M. Adams, Grant R. Zimmerman, Frederick R. Haselton, and David W. Wright

9 Gold Nanoparticle-Oligonucleotide Conjugates for the Profiling of Malignant Melanoma Phenotypes . Jobn W. Stone, Reese Harry, Owen Hendley, and David W. Wright

10 Methods for Isolating RNA Sequences Capable of Binding to or Mediating the Formation of Inorganic Materials .

Carly Jo Carter, Alina Owczarek, and Daniel L. Feldbeim 
11 Single-Walled Carbon Nanotube-Mediated Small Interfering RNA Delivery for Gastrin-Releasing Peptide Receptor Silencing in Human Neuroblastoma Jingbo Qiao, Tu Hong, Honglian Guo, ra-Qiong Xu, and Dai H. Chung

12 Amino Acid Mediated Linear Assembly of Au Nanomaterials. . . . . . . . . . . . . Manish Sethi and Marc R. Knecht

13 Enzyme-Gold Nanoparticle Bioconjugates: Quantification of Particle Stoichiometry and Enzyme Specific Activity Jacqueline D. Keighron and Christine D. Keating

14 In Vivo Testing for Gold Nanoparticle Toxicity. Carrie A. Simpson, Brian J. Huffman, and David E. Cliffel

15 Methods for Studying Toxicity of Silica-Based Nanomaterials to Living Cells

Yang Zhao, Yubui Jin, Aaron Hanson, Min Wu, and Julia Xiaojun Zhao 


\section{Contributors}

Nicholas M. Adams - Department of Chemistry, Vanderbilt University, Nashville, TN, USA Joshua M. BARnetT - Vanderbilt Eye Institute, Vanderbilt University, Nashville, TN, USA SARAH C. BAXTER - University of South Carolina, Columbia, SC, USA Carly Jo Carter • University of Colorado, Boulder, CO, USA Jerry C. Chang - Department of Chemistry, Vanderbilt University, Nashville, TN, USA DAVIN J. CherNAK - University of Illinois at Urbana-Champaign, Champaign, IL, USA Dai H. Chung - Departments of Pediatric Surgery and Cancer Biology, Vanderbilt University Medical Center, Vanderbilt University, Nashville, TN, USA

David E. Cliffel • Vanderbilt University, Nashville, TN, USA

Matthew J. Crow • Duke University, Durham, NC, USA

Daniel L. Feldheim • University of Colorado, Boulder, CO, USA

Edie C. Goldsmith • University of South Carolina, Columbia, SC, USA

Honglian Guo • Department of Electrical Engineering o Computer Science,

Vanderbilt University, Nashville, TN, USA

Aaron Hanson - University of North Dakota, Grand Forks, ND, USA

ReESE HARrY - Department of Chemistry, Vanderbilt University, Nashville, TN, USA

Frederick R. Haselton • Department of Chemistry, Vanderbilt University, Nashville,

TN, USA

Owen Hendey - Department of Chemistry, Vanderbilt University, Nashville, TN, USA

Tu Hong • Department of Electrical Engineering \& Computer Science, Vanderbilt

University, Nashville, TN, USA

Brian J. Huffman • University of North Alabama, Florence, AL, USA

Joseph A. IzaTt • Duke University, Durham, NC, USA

Ashwath Jayagopal - Vanderbilt Eye Institute, Vanderbilt University, Nashville, TN, USA YuHui Jin • University of North Dakota, Grand Forks, ND, USA

Christine D. Keating • Pennsylvania State University, University Park, PA, USA

Jacqueline D. KeIghron • Pennsylvania State University, University Park, PA, USA

MarC R. KNeCHT • University of Kentucky, Lexington, KY, USA

Joshua L. LiU • Georgia Institute of Technology, Atlanta, GA, USA

CATHERINe J. MurPhy • University of Illinois at Urbana-Champaign, Champaign,

$I L, U S A$

Alina OwczareK - University of Colorado, Boulder, CO, USA

Christine K. PAYne • Georgia Institute of Technology, Atlanta, GA, USA

John S. PENN - Vanderbilt Eye Institute, Vanderbilt University, Nashville, TN, USA

Jonas W. Perez - Department of Chemistry, Vanderbilt University, Nashville, TN, USA

Jingbo QIaO • Department of Pediatric Surgery, Vanderbilt University Medical center,

Nashville, TN, USA 
Sandra J. Rosenthal - Department of Chemistry, Vanderbilt University, Nashville, TN, USA Manish SeTHI • University of Kentucky, Lexington, KY, USA

CARrie A. Simpson • University of Colorado, Boulder, CO, USA

PATRICK N. SisCO • University of Illinois at Urbana-Champaign, Champaign, IL, USA

Melissa C. Skala - Vanderbilt University, Nashville, TN, USA

John W. STONE • Department of Chemistry, Vanderbilt University, Nashville, TN, USA

Craig J. Szymanski • Georgia Institute of Technology, Atlanta, GA, USA

Joshua R. Trantum • Vanderbilt University, Nashville, TN, USA

ADAm Wax • Duke University, Durham, NC, USA

David W. Wright • Department of Chemistry, Vanderbilt University, Nashville, TN, USA

Elizabeth R. Wright • Emory University, Atlanta, GA, USA

Min Wu • University of North Dakota, Grand Forks, ND, USA

YA-QIONG XU • Departments of Electrical Engineering \& Computer Science

and Physics \& Astronomy, Vanderbilt University, Nashville, TN, USA

Hong YI • Emory University, Atlanta, GA, USA

QI ZHANG - Pharmacology Department, Vanderbilt University, Nashville, TN, USA

Julia Xiaojun ZhaO • University of North Dakota, Grand Forks, ND, USA

YANG ZHAO - University of North Dakota, Grand Forks, ND, USA

Grant R. Zimmerman • Department of Chemistry, Vanderbilt University,

Nashville, TN, USA 